\title{
Treatment of Rockwood type III acromioclavicular joint dislocation using autogenous semitendinosus tendon graft and endobutton technique
}

This article was published in the following Dove Press journal:

Therapeutics and Clinical Risk Management

II January 2016

Number of times this article has been viewed

\author{
Gang Ye \\ Chao-An Peng \\ Hua-Bin Sun \\ Jing Xiao \\ Kang Zhu
}

Department of Orthopedics, the People's Hospital of Huangpi District, Wuhan City, People's Republic of China
Correspondence: Gang Ye

Department of Orthopedics, the People's Hospital of Huangpi District, Number 259, QianChuan, BaiXiu Street, Huangpi District, Wuhan City 430300, People's Republic of China Email yegangwh@yeah.net
Background: The aim of this study was to evaluate the therapeutic effect of autogenous semitendinosus graft and endobutton technique, and compare with hook plate in treatment of Rockwood type III acromioclavicular (AC) joint dislocation.

Methods: From April 2012 to April 2013, we treated 46 patients with Rockwood type III AC joint dislocation. Patients were randomly divided into two groups: Group A was treated using a hook plate and Group B with autogenous semitendinosus graft and endobutton technique. All participants were followed up for 12 months. Radiographic examinations were performed every 2 months postoperatively, and clinical evaluation was performed using the Constant-Murley score at the last follow-up.

Results: Results indicated that patients in Group B showed higher mean scores $(90.3 \pm 5.4)$ than Group A $(80.4 \pm 11.5)$ in terms of Constant-Murley score $(P=0.001)$. Group B patients scored higher in terms of pain $(P=0.002)$, activities $(P=0.02)$, range of motion $(P<0.001)$, and strength $(P=0.004)$. In Group A, moderate pain was reported by $2(8.7 \%)$ and mild pain by $8(34.8 \%)$ patients. Mild pain was reported by $1(4.3 \%)$ patient in Group B. All patients in Group B maintained complete reduction, while $2(8.7 \%)$ patients in Group A experienced partial reduction loss. Two patients $(8.7 \%)$ encountered acromial osteolysis on latest radiographs, with moderate shoulder pain and limited range of motion.

Conclusion: Autogenous semitendinosus graft and endobutton technique showed better results compared with the hook plate method and exhibited advantages of fewer complications such as permanent pain and acromial osteolysis.

Keywords: Rockwood type III acromioclavicular joint dislocation, autogenous semitendinosus graft, endobutton, hook plate

\section{Introduction}

Acromioclavicular (AC) joint dislocations account for $9 \%$ of shoulder girdle injuries, with a higher incidence in athletes. ${ }^{1} \mathrm{AC}$ joint dislocations are mainly caused by direct trauma, for example, a blow to the lateral shoulder pushing the acromion away from the distal clavicle. ${ }^{2}$ The AC ligament is affected first, and the effect is cascaded to the coracoclavicular (CC) ligament, and finally to the deltoid and trapezius fascia, with increasing severity. ${ }^{3}$ Therefore, treatment for $\mathrm{AC}$ joint dislocation is focused on reconstructing $\mathrm{AC}$ and $\mathrm{CC}$ ligaments.

There is no established standard of care for AC joint dislocations, although many surgical techniques have been reported. The hook plate was commonly applied in the treatment of acute AC joint dislocation with successful results. ${ }^{4-7}$ However, persistent pain, infection, delayed wound healing, arthritis, acromial impingement, and clavicle erosion and redislocation were reported in treated patients. ${ }^{7-9}$ As graft alone 
is not sufficient to prevent translation at the joint, generally a supplemental fixation is required. ${ }^{10}$ Therefore, endobutton was applied to avoid redislocation and complications.

This study presents a surgical technique using autogenous semitendinosus graft and endobutton to treat Rockwood type III AC joint dislocation. The purpose of the study was to evaluate the short-term results of the technique and compare it with the hook plate in the treatment of Rockwood type III AC joint dislocation.

\section{Materials and methods}

This was a prospective, randomized, double-blind study in a hospital setting. Patients visiting the hospital from April 2012 to April 2013 with acute type III complete AC joint dislocation were considered for inclusion in the study. Diagnosis was based on the clinical and radiological results of the patients. The study was conducted after obtaining approval from the relevant Institutional Review Board. The present study was also approved by the ethics committee of the People's Hospital of Huangpi District. Written informed consent was obtained from all patients before they entered the study.

The inclusion criteria were as follows: 1) acute type III AC joint dislocation and 2) clinical and radiological follow-up for a minimum period of 6 months. Exclusion criteria were as follows: 1) previous shoulder joint pathology on the dislocated shoulder and 2) chronic AC dislocations or other acute AC dislocations.

The patients were divided into two groups. Group A received hook plate treatment and Group B received autogenous semitendinosus graft and endobutton treatment. Randomization was performed by an independent researcher using computer-generated numbers. All investigators, research staff, and participants were blinded to the treatment allocation.

\section{Surgical technique}

In Group A, the patients underwent surgery in a beach-chair position under general anesthesia. The surgical technique was performed according to the method adopted by Wolter and Eggers. ${ }^{11}$ Removal of the hook plate was scheduled after 12 weeks.

Patient positioning in Group B were identical to the hook plate group. CC ligament was reconstructed according to the method of Struhl. ${ }^{12}$ The semitendinosus tendon harvesting was performed before AC joint exposure using a tendon stripper. The semitendinosus tendon graft was weaved and sutured with No 2 Ethibond suture (Ethicon Inc., Somerville, NJ, USA). Two drill holes were made with the diameter matching the graft from the $2.0 \mathrm{~cm}$ medial portion in the distal clavicle and on the coracoid. The prepared semitendinosus tendon was passed through two holes and sutured on the coracoid. Another drill hole was made from the $4.0 \mathrm{~cm}$ medial portion in the distal clavicle using a $2.5 \mathrm{~mm}$ drill bit. The double endobutton fixed by No 5 Ethibond sutures (Ethicon Inc.) was introduced through the third hole and the coracoid hole.

According to the postoperative protocol, the shoulder was immobilized using an abduction sling for 2 weeks. Active motion of the elbow, wrist, and hand was recommended. Passive shoulder motion was encouraged after 4 weeks postoperatively. In the follow-up period, patients were interviewed for any complaint, general physical examination was performed, and radiographs were taken. The function of the repaired joint was evaluated using the Constant-Murley scale at the last follow-up.

\section{Functional outcomes}

According to the Constant-Murley score, ${ }^{13}$ four variables were used to assess the function of the shoulder. The two subjective variables included pain and limitation of activities of daily living, and the two objective variables included range of motion and strength of the shoulder. The subjective part of the test was self-completed by the patient. The absence of pain was allocated a maximum score of 15 ; no limitation of activities of daily living like sleep, work, recreation, or sport, was allocated a maximum score of 20. Range of motion (maximum 40 points) and strength (maximum 25 points) were measured.

\section{Radiographic outcomes}

Radiographs of the AC joint were obtained every 2 months postoperatively. The radiographic studies were examined for signs of loss of reduction, or other complications such as fracture or distal clavicular osteolysis. The maintenance of $\mathrm{AC}$ joint reduction was defined as follows: 1) maintained reduction, no side-to-side difference on anteroposterior (AP) radiographs; 2) partial loss of reduction, a side-to-side difference of less than the width of the clavicle; and 3) complete loss of reduction, a side-to-side difference in excess of the clavicle width. ${ }^{14}$

\section{Statistical analysis}

Categorical and continuous variables were described (ratios, means, and standard deviations). The statistical analyses of differences in continuous variables between the two groups were tested with unpaired Student's $t$-test and differences in categorical variables with $\chi^{2}$ test. Nonparametric tests 
of K-independent samples were performed on the equality of the medians for the continuous variables without normal distribution. All analyses were performed with SPSS version 21.0 (SPSS, Chicago, IL, USA). Differences were considered significant at $P<0.05$.

\section{Results}

Forty-six patients (30 men, 16 women) were included in the study. Table 1 shows the baseline characteristics of the patients. The mean age of the participants was 33.4 years (range 30-46 years) at enrollment. The treatment groups were well balanced in terms of baseline characteristics.

\section{Clinical outcomes}

Clinical evaluation was performed using the ConstantMurley score ${ }^{13}$ at the final follow-up. The patients acquired an average of 90.3 points (maximum 100 points) in Group B, better than 80.4 points in Group A in the Constant-Murley score $(P<0.05)$. Table 2 shows that patients treated with the autogenous semitendinosus graft and endobutton technique showed higher constant scores than the hook plate-treated patients with regard to pain $(P=0.002)$, activities $(P=0.02)$, range of motion $(P<0.001)$, and strength $(P=0.004)$. During the follow-up period, moderate pain was reported by two $(8.7 \%)$ hook plate-treated patients and mild pain was reported by eight (34.8\%) hook plate-treated patients. Mild pain was reported by one $(4.3 \%)$ patient treated with autogenous semitendinosus graft and endobutton technique.

\section{Radiographic finding}

On final follow-up, all the patients of Group A maintained complete reduction in the AP plane, except $2(8.7 \%)$ who experienced partial reduction loss. None of the patients experienced complete loss of reduction. There were 2 (8.7\%) patients in Group A with acromial osteolysis on the latest radiographs, which included a 38-year-old woman and a 45-year-old man (Figure 1A and B). Their constant scores were 59 and 55, respectively. Moderate shoulder pain and limited range of motion were reported by both of them. There

Table I Baseline characteristics of patients

\begin{tabular}{llll}
\hline & $\begin{array}{l}\text { Group A } \\
(\mathbf{n}=\mathbf{2 3})\end{array}$ & $\begin{array}{l}\text { Group B } \\
(\mathbf{n = 2 3 )}\end{array}$ & P-value \\
\hline Sex (men/women) & $14 / 9$ & $16 / 7$ & 0.76 \\
Age (years), mean (SD) & $33.4(3.3)$ & $34.3(3.3)$ & 0.40 \\
Time from injury to & $3.4(0.7)$ & $3.5(0.7)$ & 0.69 \\
operation (days), mean (SD) & & & \\
Injury side (n), (right/left) & $\mathrm{II//2}$ & $9 / 14$ & 0.35 \\
\hline
\end{tabular}

Table 2 Evaluation results of patients at the last follow-up according to the Constant-Murley score (n [\%])

\begin{tabular}{llll}
\hline & Group A & Group B & P-value \\
\hline Pain & $15.0(5.0,15.0)$ & $10.0(5.0,15.0)$ & 0.002 \\
Activities & $18.4 \pm 2.1$ & $19.6 \pm 0.8$ & 0.02 \\
Range of motion & $33.0 \pm 3.5$ & $37.0 \pm 2.0$ & $<0.001$ \\
Strength & $16.5 \pm 3.2$ & $18.9 \pm 2.1$ & 0.004 \\
Total & $80.4 \pm 11.5$ & $90.3 \pm 5.4$ & 0.001 \\
\hline
\end{tabular}

Note: Data presented as constant scores: either as mean \pm SD or median (quartiles).

were no infections or fractures in patients who were included in the study. However, all patients in Group B maintained complete reduction (data not shown).

\section{Discussion}

$\mathrm{AC}$ joint is an articulation of the lateral clavicle and the acromion. As an amphiarthrodial joint, it plays an important role in maintaining the strength and movement of the whole upper limb. Garretson and Williams ${ }^{2}$ classified the degree of displacement in $\mathrm{AC}$ joint dislocations into six types based on the severity of involvement of $\mathrm{AC}$ and $\mathrm{CC}$ ligaments. The initial treatment of an acute type III dislocation of AC joint remains controversial. ${ }^{6,15-17}$ Though the surgical treatments have improved in recent years, a high incidence of complications has also been reported in some studies, and thereby failing to establish a gold standard. However, in our clinical study, we found that the surgical treatment always receives the better effects compared to the conservative treatment, according to our 5 years of follow-up. Therefore, we explored the therapeutic effects of surgical treatment over the conservative treatment.

In this study, we evaluated the effect of surgery using autogenous semitendinosus graft and endobutton technique for the treatment of Rockwood type III AC joint dislocation. The previous studies and our clinical practice have been indicated that neither the autogenous semitendinosus graft technique nor the endobutton technique can therapy the Rockwood type III AC joint dislocation efficiently. ${ }^{6,18-22}$ Therefore, in this study, we added the semitendinosus graft to the endobutton technique. Furthermore, we also observed several types of dislocations in clinical, however, therapy of the type III dislocation with the best outcomes. Therefore, we selected the type III dislocation as the study type.

Higher mean constant score was obtained in patients treated using this technique (90.3 points) compared with hook plate-treated patients (80.4 points). Radiographic findings showed that all patients in Group B achieved reconstruction in the AP plane anatomically, while $8.7 \%$ (2 of 23 ) patients 

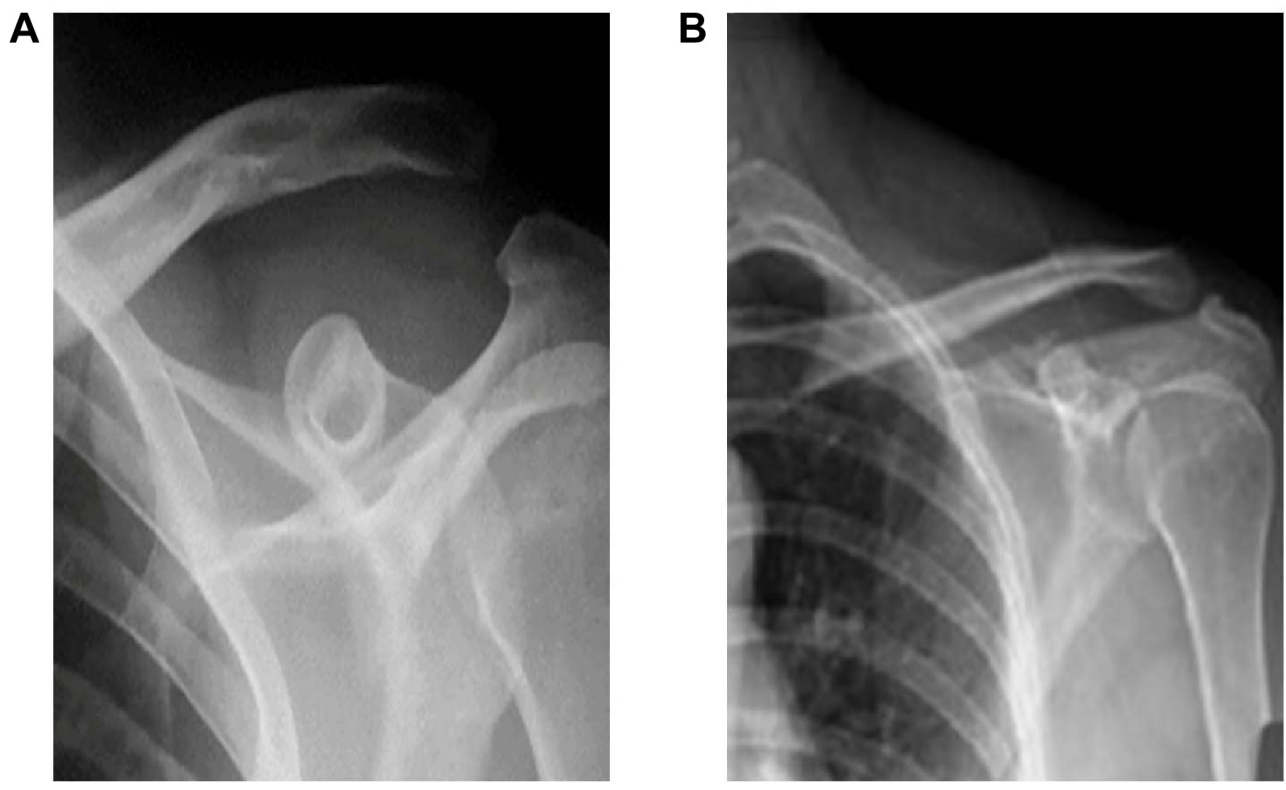

Figure I Radiographic finding of acromial osteolysis patients.

Notes: (A) The radiograph of the 38-year-old woman. (B) The radiograph of the 45-year-old man.

in Group A experienced partial reduction loss. Moreover, two cases $(8.7 \%)$ of acromial osteolysis with moderate shoulder pain and limited range of motion were observed in Group A. Gstettner et $\mathrm{al}^{6}$ reported a high possibility of subluxation in $24.7 \%$ of patients using a modified endobutton technique compared with our study. This may be probably because of the fact that autogenous semitendinosus graft was applied together with endobutton.

Martetschlager et $\mathrm{al}^{18}$ reported four cases of graft ruptures in 46 shoulders using the tendon graft technique. The four graft ruptures encountered were associated with the insufficient strength of the graft. Mazzocca et al ${ }^{19}$ suggested that autograft is not sufficient to prevent anterior-posterior translation at the joint. Therefore, modifications to augment the autograft with a fixation material around the base of the coracoid process were essential to prevent graft ruptures.

In another study using a double-button and fixation technique, Milewski et $\mathrm{al}^{20}$ reported ten cases involving shuttling the graft through an additional tunnel in the coracoid base. In addition to the chronic dislocations included in the series, a higher rate of coracoid fractures associated with coracoid tunnel techniques indicated that modifications, including the location and diameter of drill holes, should be considered to prevent fractures. In our study, the diameter of the hole matched with the graft to avoid the fractures.

Currently, endobutton and hook plate are widely used. The mobile side of the hook plate underneath the acromion may cause symptoms of persistent pain and restriction in movement by acromial erosion and damaging the supraspinatus tendon. ${ }^{9,21}$ Long-term retention of the plate may lead to acromial osteolysis or fracture, which implies that a second surgery is required to remove the plate after 12 weeks when the ligaments are healed. Kienast et $\mathrm{al}^{7}$ achieved successful results using the hook plate in 313 patients of Rockwood type III-V AC joint dislocation, while all patients reported pain or discomfort with the hook plate. After removal of the hook plate, a high incidence of complications of superficial soft tissue infections and fracture of the acromion and redislocations were reported.

Endobutton is an appropriate technique to reproduce the course of the conoid portion of the CC ligament. The technique was first introduced by Struhl. He had performed only a small incision with minimal soft tissue dissection. The strength and stiffness of the device exceeded the native ligament complex by approximately $40 \%{ }^{12}$ The deforming forces are distributed on the surface of the double endobutton plates, thereby reducing the effect on the suture material and minimizing the chance of soft tissue reaction to the suture material. The modified device added a second endobutton to create a knotless fixation. The objective was to prevent complications, especially shoulder pain by minimizing recurrent displacement and joint irritation, and to avoid soft tissue reactions and traumatic arthritis. ${ }^{19,21}$ Because of the biocompatibility of the device, there is no need to remove the device in a second surgery, which can avoid potential injuries. $^{22}$

This study demonstrated the open technique and exhibited advantages of few complications such as permanent 
pain and acromial osteolysis. During the surgery, attention should be paid to the diameter and location of the hole during drilling. Minor diameter and accurate location are of paramount importance. To achieve better stability, the distance of the insertion site of the clavicle and acromion should be approximate to the anatomical position of 5.2 $\mathrm{mm}$ (women) and $7.6 \mathrm{~mm}$ (men). ${ }^{23}$ Future studies are warranted to explore the long-term therapeutic results of this technique.

Actually, the other factors could also affect the clinical outcomes, such as rehab. The limitation of this work is that it only investigated the single-factor-dependent clinical outcomes. Therefore, in the following studies, we would also explore the effects of rehab on the clinical outcomes.

\section{Conclusion}

This described technique shows better short-term results than the hook plate method and can be used effectively in the treatment of Rockwood type III AC dislocation.

\section{Disclosure}

The authors report no conflicts of interest in this work.

\section{References}

1. McCreesh KM, Riley SJ, Crotty JM. Acromio-clavicular joint cyst associated with a complete rotator cuff tear: a case report. Man Ther. 2014; 19(5):490-493.

2. Garretson RB, Williams GR. Clinical evaluation of injuries to the acromioclavicular and sternoclavicular joints. Clin Sports Med. 2003;22(2): 239-254.

3. Mazzocca A, Arciero R, Bicos J. Evaluation and treatment of acromioclavicular joint injuries. Am J Sports Med. 2007;35(2):316-329.

4. Ejam S, Lind T, Falkenberg B. Surgical treatment of acute and chronic acromioclavicular dislocation Tossy type III and V using the Hook plate. Acta Orthop Belg. 2008;74(4):441-445.

5. Di Francesco A, Zoccali C, Colafarina O, Pizzoferrato R, Flamini S. The use of hook plate in type III and V acromio-clavicular Rockwood dislocations: clinical and radiological midterm results and MRI evaluation in 42 patients. Injury. 2012;43(2):147-152.

6. Gstettner C, Tauber M, Hitzl W, Resch H. Rockwood type III acromioclavicular dislocation: surgical versus conservative treatment. J Shoulder Elbow Surg. 2008;17(2):220-225.
7. Kienast B, Thietje R, Queitsch C, Gille J, Schulz AP, Meiners J. Midterm results after operative treatment of Rockwood grade III-V acromioclavicular joint dislocations with an AC-hook-plate. Eur JMed Res. 2011; 16(2):52-56.

8. Chiang CL, Yang SW, Tsai MY, Kuen-Huang Chen C. Acromion osteolysis and fracture after hook plate fixation for acromioclavicular joint dislocation: a case report. J Shoulder Elbow Surg. 2010;19(4):e13-e15.

9. Chandrasenan J, Badhe S, Cresswell T. The clavicular hook plate: consequences in three cases. Eur J Trauma Emerg Surg. 2007;33(5):557.

10. Weinstein DM, McCann PD, McIlveen SJ, Flatow EL, Bigliani LU. Surgical treatment of complete acromioclavicular dislocations. $\mathrm{Am}$ J Sports Med. 1995;23(3):324-331.

11. Wolter D, Eggers C. Reposition and fixation of acromioclavicular luxation using a hooked plate. Hefte Unfallheilkd. 1984;170(1):80-86.

12. Struhl S. Double endobutton technique for repair of complete acromioclavicular joint dislocations. Tech Shoulder Elbow Surg. 2007;8(2): 175-179.

13. Karlsson J, Árnarson H, Sigurjónsson K. Acromioclavicular dislocations treated by coracoacromial ligament transfer. Arch Orth Trauma Surg. 1986;106(1):8-11.

14. Jiang C, Wang M, Rong G. Proximally based conjoined tendontransfer for coracoclavicular reconstruction in the treatment of acromioclavicular dislocation. J Bone Joint Surg Am. 2007;89(11):2408-2412.

15. Broos P, Stoffelen D, Van de Sijpe K, Foumeau I. Surgical management of complete Tossy III acromioclavicular joint dislocation with the Bosworth screw or the Wolter plate. A critical evaluation. Unfallchirurgie. 1997;23(4):153-159.

16. Larsen E, Bjerg-Nielsen A, Christensen P. Conservative or surgical treatment of acromioclavicular dislocation. A prospective, controlled, randomized study. J Bone Joint Surg Am. 1986;68(4):552-555.

17. Galpin RD, Hawkins RJ, Grainger RW. A comparative analysis of operative versus nonoperative treatment of grade III acromioclavicular separations. Clinical Orthop Relat Res.1985;193(1):150-155.

18. Martetschlager F, Horan MP, Warth RJ, Millett PJ. Complications after anatomic fixation and reconstruction of the coracoclavicular ligaments. Am J Sports Med. 2013;41(12):2896-2903.

19. Mazzocca AD, Santangelo SA, Johnson ST, Rios CG, Dumonski ML, Arciero RA. A biomechanical evaluation of an anatomical coracoclavicular ligament reconstruction. Am J Sports Med. 2006;34(2):236-246.

20. Milewski MD, Tompkins M, Giugale JM, Carson EW, Miller MD, Diduch DR. Complications related to anatomic reconstruction of the coracoclavicular ligaments. Am J Sports Med. 2012;40(7):1628-1634.

21. Li H, Wang C, Wang J, Wu K, Hang D. Restoration of horizontal stability in complete acromioclavicular joint separations: surgical technique and preliminary results. Eur J Med Res. 2013;18(1):42.

22. Lu JW, Song HP, Lin BY, Lu D, Liu H. Improvement technique of double endobutton plate for the treatment of type III acromioclavicular joint dislocation. Zhongguo Gu Shang. 2010;23(11):865-867.

23. Renfree K, Riley M, Wheeler D, Hentz JG, Wright TW. Ligamentous anatomy of the distal clavicle. J Shoulder Elbow Surg. 2003;12(4): 355-359.
Therapeutics and Clinical Risk Management

\section{Publish your work in this journal}

Therapeutics and Clinical Risk Management is an international, peerreviewed journal of clinical therapeutics and risk management, focusing on concise rapid reporting of clinical studies in all therapeutic areas, outcomes, safety, and programs for the effective, safe, and sustained use of medicines. This journal is indexed on PubMed Central, CAS,

\section{Dovepress}

EMBase, Scopus and the Elsevier Bibliographic databases. The manuscript management system is completely online and includes a very quick and fair peer-review system, which is all easy to use. Visit $\mathrm{http}: / / \mathrm{ww}$.dovepress.com/testimonials.php to read real quotes from published authors. 